\title{
On the issue of choosing a real-time operating system for hardware and software support of industrial and environmental monitoring systems
}

\author{
D.I. Kovalev ${ }^{1,2,4}$, T.P. Mansurova ${ }^{2}$, Ya.A. Tynchenko ${ }^{3}$ \\ ${ }^{1}$ Krasnoyarsk State Agrarian University, 90, Mira pr., Krasnoyarsk, 660049, Russia \\ ${ }^{2}$ Krasnoyarsk Science and Technology City Hall of the Russian Union of Scientific and \\ Engineering Association, 61, Uritskogo Str., Krasnoyarsk, 660049, Russia \\ ${ }^{3}$ Reshetnev Siberian State University of Science and Technology, 31, Krasnoyarsky Rabochy \\ Av., Krasnoyarsk, 660037, Russia \\ ${ }^{4}$ E-mail: grimm7jow@gmail.com
}

\begin{abstract}
The article discusses the features of the operation of environmental monitoring systems. A number of factors are determined that affect the organization of hardware and software support for such systems. The main factors are: the operation of the equipment in real time, various environmental influences, the specifics of the composition of sensors and connections, as well as a complex technology for servicing the equipment in an aggressive technological environment. The listed factors affect the structure, software, hardware components and design of environmental monitoring systems. When creating any complex systems, the distribution of work in time leads to the allocation of various design stages, and the ideas about the system being designed, reflecting its essential properties with varying degrees of detail, determine the constituent parts of the design process. The specificity of the stages for monitoring systems of thermal power plants is shown, determined both by the general features of these systems and by the peculiarities of their application in the technological processes of thermal power plants, among which the main ones are: the heterogeneity of the input units and devices, as well as technological objects of power plants; work in real time; programmable logic of smart sensors and multifunctional purpose of system components; the possibility of failures leading to a change in the functioning algorithm using the multiversion methodology; the presence of interrelated requirements for the accuracy and speed of information transfer. The work shows the content of work on the stages of designing monitoring systems, taking into account the specifics of their development. This specificity is most significantly manifested in the development of a technical proposal and a draft design. In a step-bystep design, after the end of each stage, an assessment of the main results obtained is carried out by comparing them with those required according to the terms of reference. The article presents an expert analysis that allows you to determine the acceptable implementation of a real-time operating system for hardware and software support for environmental monitoring technologies. Phar Lap ETS, VxWorks and NI Linux Real-Time operating systems are considered.
\end{abstract}

Keywords: environmental monitoring, power plant, real-time operating system, software, technological process 
Современные инновачии, системы и технологии, 2021, 1(2)

УДК 004.032.2

\title{
К вопросу выбора операционной системы реального времени для аппаратно-программной поддержки систем производственно-экологического мониторинга
}

\author{
Д.И. Ковалев ${ }^{1,2,4}$, Т.П. Мансурова ${ }^{2}$, Я.А. Тынченко ${ }^{3}$ \\ ${ }^{1}$ Красноярский государственный аграрный университет, пр. Мира, 90, Красноярск, \\ 660049, Россия \\ 2 Красноярский краевой Дом науки и техники Российского Союза научных и \\ инженерных общественных объединений, ул. Урицкого, 61, Красноярск, 660049, Россия \\ ${ }^{3}$ Сибирский государственный университет науки и технологий имени академика \\ М.Ф. Решетнева, пр. Красноярский рабочий, 31, Красноярск, 660037, Россия \\ ${ }^{4}$ E-mail: grimm7jow@gmail.com
}

\begin{abstract}
Абстракт. В статье рассматриваются особенности эксплуатации систем экологического мониторинга. Определяется ряд факторов, влияющих на организацию аппаратно-программной поддержки таких систем. Основными факторами являются: работа аппаратуры в реальном масштабе времени, различные воздействия окружающей среды, специфика состава датчиков и связей, а также сложная технология обслуживания аппаратуры в агрессивной технологической среде. Перечисленные факторы влияют на структуру, программное обеспечение, элементную базу и конструкцию систем экологического мониторинга. При создании любых сложных систем распределение работ во времени приводит к выделению различных этапов проектирования, а представления о проектируемой системе, отражающие ее существенные свойства с той или иной степенью подробности, определяют составные части процесса проектирования. Показана специфика этапов для систем мониторинга тепловых электростанций, определяемая как общими особенностями этих систем, так и особенностями их применения в технологических процессах тепловых электростанций, среди которых главными являются: разнородность входящих блоков и устройств, а также технологических объектов электростанций; работа в реальном масштабе времени; программируемая логика работы интеллектуальных датчиков и многофункциональное назначение компонентов системы; возможность возникновения отказов, приводящих к изменению алгоритма функционирования, с использованием мультиверсионной методологии; наличие взаимосвязанных требований по точности и скорости передачи информации. В работе показано содержание работ по этапам проектирования систем мониторинга с учетом специфики их разработки. Эта специфика наиболее существенно проявляется при разработке технического предложения и эскизного проекта. При поэтапном проектировании после окончания каждого этапа выполняется оценка основных полученных результатов путем сравнения их с требуемыми по техническому заданию. В статье представлен экспертный анализ, который позволяет определить допустимый вариант реализации операционной системы реального времени для аппаратно-программной поддержки технологий экологического мониторинга. Рассмотрены операционные системы реального времени Phar Lap ETS, VxWorks и NI Linux Real-Time.
\end{abstract}

Ключевые слова: экологический мониторинг, электростанция, операционная система реального времени, программное обеспечение, технологический процесс 


\section{1. Введение}

Окружающая среда, влияя на выбор элементной базы, построение структуры и создание конструкции систем производственно-экологического мониторинга (СПЭМ), определяется многими факторами, среди которых, в первую очередь, следует учитывать климатические условия, механические, тепловые, химические, радиационные воздействия [1$6]$.

Основными климатическими воздействиями принято считать изменения температуры и относительной влажности. Диапазон изменения рабочих температур для аппаратуры прежде всего определяется климатическими зонами Земли, в которых возможно использование разрабатываемой техники. Поскольку измерительная аппаратура (датчики) может эксплуатироваться фактически в любых климатических зонах, диапазон рабочих температур должен учитывать предельные температуры среды в различных точках технологического объекта, на котором устанавливается СПЭМ.

Решение задач в реальном масштабе времени в контуре мониторинга определяется необходимостью обеспечения заданного функционала системы [4]. Для эффективного функционирования аппаратно-программного комплекса (АПК) системы обмена информацией должна иметь архитектуру, обеспечивающую работу в реальном времени. Это означает, что структурная схема и программное обеспечение СПЭМ необходимо проектировать с учетом этого требования. При этом серьезное внимание следует обратить на адекватность используемых алгоритмов решения задач обработки информации и управления АПК требованию реального времени. А это существенно зависит от выбранной операционной системы реального времени (ОСРВ).

\section{2. Постановка задачи}

Для обоснованного анализа требований к современным СПЭМ необходимо четко представлять весь комплекс их технических характеристик. Все множество X технических характеристик СПЭМ можно представить следующим образом [7]:

$$
\mathrm{X}=\left\{\mathrm{X}_{\text {обслл }} ; \mathrm{X}_{\text {стр }} ; \mathrm{X}_{\text {по }} ; \mathrm{X}_{\text {эк }} ; \mathrm{X}_{\text {кн }}\right\} \text {, }
$$

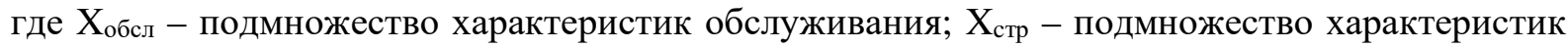
структур; $\mathrm{X}_{\text {по }}$ - подмножество характеристик программного обеспечения; $\mathrm{X}_{\text {эк }}$ - подмножество характеристик условий эксплуатации; $\mathrm{X}_{\mathrm{\kappa н}}$ - подмножество характеристик конструкции.

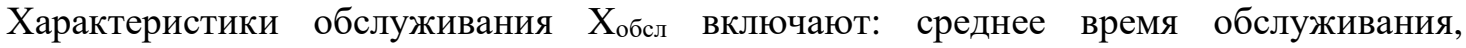
вероятность обслуживания требования на обмен, среднее время простоя канала обмена, 
вероятность простоя канала, вероятность нахождения системы обмена в одном из рабочих состояний.

Структура СПЭМ определяется подмножеством характеристик $\mathrm{X}_{\text {стр }}$, зависящим от типа, вида и режима обмена; числа каналов обмена; способа передачи кодов и их типа; типа управления; параметров контроля; программируемости, адаптивности, перестраиваемости; надежности функционирования и собственно аппаратной; пропускной способности; числа абонентов; типа интерфейса; электрических характеристик входных и выходных сигналов канала обмена; типа используемой элементной базы.

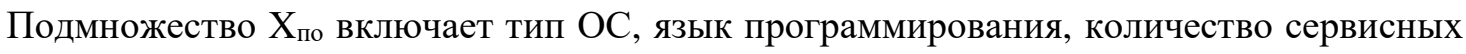
и стандартных подпрограмм, объем памяти, необходимой для хранения ПО.

Условия эксплуатации (подмножество $\mathrm{X}_{\text {эк }}$ определяются параметрами среды и первичной сети энергопитания, временем непрерывной работы, техническим ресурсом, параметрами обдува или микроклимата, временем готовности к работе.

Характеристики конструкции (подмножество $\mathrm{X}_{\mathrm{\kappa H}}$ ) весьма обширны, но можно ограничиться следующей совокупностью параметров: массогабаритные характеристики, тип конструкции, энергопотребление, коэффициенты технологичности, унификации и стандартизации, содержание драгоценных металлов, ремонтоспособность, стоимость и экономическая эффективность.

Десятки указанных выше характеристик полностью определяют облик СПЭМ, но на практике редко используются вместе. В минимальную совокупность технических характеристик $\mathrm{X}_{\min }$, с достаточной для практики достоверностью и определенностью обрисовывающую облик СПЭМ, целесообразно включить ограниченное число параметров:

$$
\mathrm{X}_{\min }=\left\{\mathrm{T}_{\mathrm{obs}} ; \mathrm{P}_{\mathrm{str}} ; \mathrm{M}_{\mathrm{c}} ; \mathrm{C}_{\mathrm{pr}} ; \mathrm{P}_{\mathrm{nr}} ; \mathrm{mA} ; \mathrm{L}_{\mathrm{p}} ; \mathrm{X}_{\mathrm{cp}} ; \mathrm{G}_{\mathrm{a}} ; \mathrm{W} ; \mathrm{C}_{\mathrm{a}}\right\}
$$

где $\mathrm{T}_{\mathrm{obs}}-$ среднее время обслуживания, мкс;

$\mathrm{P}_{\mathrm{str}}$ - вероятности нахождения системы в рабочих состояниях;

$\mathrm{M}_{\mathrm{c}}$ - тип, вид и режим обмена (Мт, Мв, Мр);

$\mathrm{C}_{\mathrm{pr}}$ - пропускная способность, кб/с;

$\mathrm{P}_{\mathrm{nr}}$ - вероятность нормального функционирования;

$\mathrm{mA}$ - число обслуживаемых датчиков;

Lp - язык программирования;

$\mathrm{X}_{\mathrm{cp}}$ - параметры окружающей среды;

$\mathrm{G}_{\mathrm{a}}-$ масса, кг;

$\mathrm{W}$ - энергопотребление, Вт;

$\mathrm{C}_{\mathrm{a}}$ - стоимость. 
Для ориентировочной оценки СПЭМ и сравнения различных реализаций обычно используют еще более сокращенный перечень технических характеристик, а именно: $\mathrm{M}_{\mathrm{c}}, \mathrm{C}_{\mathrm{pr}}$, $\mathrm{mA}, \mathrm{G}_{\mathrm{a}}$.

\section{3. Методы решения с учетом эксплуатационных характеристик СПЭМ}

\section{1. Особенности эксплуатации}

Рассмотрим особенности эксплуатации СПЭМ. Для измерительной электронной аппаратуры датчиков различного применения основные механические факторы проявляются в виде вибрационных воздействий и ударов вибрации с частотой от 2 до 7000 Гц при ускорениях от долей $\mathrm{g}$ до $50 \mathrm{~g}$; удары от $65 \mathrm{~g}$ при длительностях импульсов в несколько миллисекунд (в среднем 10мс).

Влияние радиационного излучения на микроэлектронную аппаратуру измерительных устройств аналогично его влиянию на радиоэлектронную аппаратуру вообще. При действии радиации ухудшаются практически все характеристики аппаратуры, но в настоящее время уже созданы комплекты измерительных датчиков, нормально функционирующих при достаточно больших интегральных дозах облучения - до 5000 рад.

Хранение бортовой аппаратуры должно быть допустимо на складах любого типа (открытые, закрытые, отапливаемые и без отопления), а транспортирование должно обеспечиваться любым видом транспорта на любые расстояния. Имеющиеся при этом ограничения по температуре, вибрациям, ударам и радиации определяются видом аппаратуры, применением, а номинальные воздействия ограничены указанными выше диапазонами.

Факторы воздействия окружающей среды определяют требования, которым должны соответствовать условия эксплуатации применяемой элементной базы и материалов конструкции [6]. Кроме того, технология создания конструкции, а также заложенные разработчиками конструктивные решения должны обеспечивать нормальное функционирование аппаратуры в условиях окружающей среды. При создании структуры СПЭМ должны быть предусмотрены технические решения, гарантирующие работоспособность системы обмена данными при деградационных изменениях параметров элементной базы под действием температуры, влажности, вибрации, ударов, радиации и т.д. [7]. Например, использование различных устройств, имеющих разную степень деградации, а также применение аппаратной и программной избыточности для переключения приборов при отказах и повышения информационной надежности СПЭМ. 


\section{2. Состав датчиков и обмен данными}

При рассмотрении специфики состава датчиков и связей между ними для АПК в целом, что существенно влияет на построение СПЭМ, в первую очередь необходимо учитывать: число датчиков и их разнородность, число и протяженность линий связи, топологию размещения датчиков и линий связи на технологическом объекте, а также влияние помех на передаваемые сигналы.

На современных объектах мониторинга (включая объекты теплоэнергетики [8-10]) в рамках АПК осуществляется подключение сотен датчиков (обычно 50-250 при максимальном числе 1000). Датчики принимают и выдают информацию в различной форме в широком диапазоне значений передаваемых величин с различной погрешностью. Обобщая данные различных датчиков объектов мониторинга для различных областей применения, можно представить усредненные параметры всей совокупности датчиков.

Следует кратко остановиться еще на одной характеристике интеллектуальных датчиков, отражающей их «интеллект», - способность вести диалог с системой управления или оператором. В общем случае под «интеллектуальными» понимают такие датчики, которые могут адаптироваться вплоть до передачи информации на естественном языке. Разработки таких датчиков и их интерфейсов ведутся в настоящее время в различных странах.

В качестве линий связи на объектах применяются отдельные провода, шины и кабели. Наиболее перспективны магистральные мультиплексные шины - волоконно-оптические линии связи (ВОЛС). Число линий связи на объектах достаточно велико. Часто для передачи данных могут использоваться спутниковые каналы [11-13]. Особенно это актуально для мониторинга потенциально опасных технологических объектов или в случае чрезвычайной ситуации на таком объекте [14-16].

Специфика состава датчиков и линий связи непосредственно влияет на все компоненты СПЭМ, а именно:

- используемая элементная база должна обеспечивать требуемые скоростные, точностные, надежностные и эксплуатационные характеристики сигналов датчиков и соответствующие параметры;

- структура системы обмена данными должна включать: блоки интерфейсов, соответствующие датчикам и связям; устройства преобразования формы представления сигналов (аналог - код, код - аналог, код - код); устройства связи с магистралями, мультиплексоры, модемы, а также блоки организации диалога с «интеллектуальными» датчиками и блоки согласования скорости передачи информации со скоростными возможностями управляющей ЭВМ при решении 
задач СПЭМ (необходимо учитывать в структуре требуемые типы, виды и режимы обмена с датчиками, возможность адаптации под любой тип датчика);

- программное обеспечение СПЭМ должно организовывать работу всех каналов в соответствии с используемыми методами обмена, алгоритмами управления, с учетом характеристик датчиков, выдающих исходные данные и отрабатывающих результаты вычислений ЭВМ;

- в конструкции СПЭМ должны быть предусмотрены необходимые коммутационноустановочные элементы, сопряженные с используемыми линиями связи (состав, топология, тип) и обеспечивающие имеющиеся требования по защите от помех; конструкция также должна удовлетворять требованию быстрой замены интерфейсных блоков при изменении используемых стандартов на линии связи или состава абонентов; конструктивные решения должны быть инвариантны к топологии размещения датчиков СПЭМ и аппаратуры самой СПЭМ.

\section{3. Требования к надежности аппаратуры}

Организация обслуживания СПЭМ наиболее существенно зависит от требуемой надежности аппаратуры, состава ЗИПа, структуры контрольно-проверочной (КПА) и контрольно-ремонтной (КРА) аппаратуры, параметров ресурса, хранения, транспортировки, а также квалификации обслуживающего персонала [7]. Все отмеченные факторы, с одной стороны, определяются созданной СПЭМ, а с другой стороны, сами влияют на структуру, конструкцию, программно-аппаратное обеспечение СПЭМ. Так, надежность системы определяет порядок и периодичность обслуживания, но само обслуживание обеспечивает поддержание заданной надежности структуры АПК и ПО СПЭМ на соответствующем уровне системы. Созданные или имеющиеся возможности организации обслуживания влияют на выбор элементной базы, ограничивают сложность разрабатываемой архитектуры и оригинальность конструкторских решений.

\section{4. Факторы обслуживания СПЭМ}

Рассмотрим подробнее факторы, осложняющие обслуживание СПЭМ. Структура современных СПЭМ очень сложна как по функциональным связям, так и по схемноструктурным решениям. Применение микропроцессоров, мультиобработки, шинных мультиплексных связей, специализированных процессоров обмена, выполнение разнообразных функций обуславливают повышенную сложность архитектуры. Все отмеченное требует научной организации обслуживания с использованием современной 
универсальной и специализированной измерительной техники, а также непрерывного повышения квалификации соответствующего персонала.

Программное обеспечение, являясь важнейшей частью архитектуры современной СПЭМ и влияя на организацию обслуживания, требует расширения парка необходимой аппаратуры и номенклатуры работников этих служб. Применение языков высокого уровня (ЯВУ), развитых операционных систем реального времени (ОСРВ), микропрограммирования обуславливает наличие резидентных и кросс-средств отладки рабочих программ, участие в обслуживании системных программистов и специалистов по вычислительной математике [8].

Для осуществления высокоэффективного обслуживания, обеспечивающего нормальное функционирование СПЭМ при заданном ресурсе эксплуатации, конструкторскотехнологические решения, заложенные в систему обмена данными, должны обеспечивать: требуемую защиту аппаратуры от воздействий окружающей среды; заданную надежность; блочную организацию ЗИПа; быструю замену неисправных элементов; высокую технологичность всех проверок и ремонтов; требуемую помехозащищенность электрических схем; заданные массогабаритные характеристики при выполнении отраслевых норм на коэффициенты унификации и стандартизации; необходимые условия хранения и транспортировки аппаратуры.

Основные приборы, оборудование, приспособления и инструменты, применяемые при обслуживании, сосредоточены в составе КПА и КРА систем обмена. Эта аппаратура должна учитывать новые решения по элементной базе, структуре, ПО и конструкции. Это приводит к необходимости использовать при проверках и ремонтах автоматизированную аппаратуру на базе специализированных ЭВМ, а также современное технологическое оборудование. Все это также предопределяет участие различных специалистов высокой квалификации.

Рассмотренные особенности эксплуатации, их взаимосвязь с используемой элементной базой, архитектурой и конструкцией СПЭМ необходимо учитывать при проектировании систем обмена информацией с целью повышения информационной надежности технологий экологического мониторинга.

\section{4. Учет особенностей проектирования СПЭМ}

При создании любых сложных систем распределение работ во времени приводит к выделению различных этапов проектирования, а представления о проектируемой системе, отражающие ее существенные свойства с той или иной степенью подробности, определяют составные части процесса проектирования [7]. Это уровни проектирования.

В качестве типовых этапов проектирования используются: 
Modern Innovations, Systems and Technologies, 2021, 1(2)

• этап научно-исследовательских работ (технического предложения),
заканчивающийся формулированием принципиальных возможностей построения
системы, а также определением основных элементов структуры и ПО, физических
и технических свойств проектируемой системы и т.п.;
• этап эскизного проектирования, на котором детально прорабатывается
возможность построения системы и выполняется эскизный проект;
• этап технического (рабочего) проектирования, в процессе выполнения которого
разрабатывается технический проект, где тщательно прорабатываются все
функциональные, схемные, конструкторские и технологические решения с
разработкой соответствующей документации;
этап рабочих испытаний, на котором производится окончательная проверка
выбранных решений по системе, а также оформляются протоколы испытаний
СпЭМ.

Работы на этапах проектирования по форме аналогичны для всех типов систем мониторинга. Однако есть специфика этапов для СПЭМ, определяемая общими особенностями этих систем и особенностями их применения в технологических процессах ТЭС, среди которых главными являются:

- разнородность входящих блоков и устройств, а также технологических объектов ТЭС;

- работа в реальном масштабе времени;

- программируемая логика работы интеллектуальных датчиков и многофункциональное назначение компонентов системы (например, различные типы газоанализаторов);

- возможность возникновения отказов, приводящих к изменению алгоритма функционирования при отказах элементов (использование мультиверсионной методологии в СПЭМ);

- наличие взаимосвязанных требований по точности и скорости передачи информации.

В таблице 1 показано содержание работ по этапам проектирования СПЭМ с учетом специфики их разработки. Эта специфика наиболее существенно проявляется при разработке технического предложения и эскизного проекта. 
Современные инновации, системы и технологии, 2021, 1(2)

Таблица 1. Содержание работ на этапах проектирования.

Этап проектирования

Техническое предложение

Эскизное проектирование

Техническое проектирование

Испытания
Выполняемая работа

- Определение предложений в архитектуре.

- Исследование возможностей реализации связей со всеми разнотипными абонентами СПЭМ.

- Оценочный анализ влияния всех видов отказов на основные характеристики системы.

- Поиск путей выполнения требований к характеристикам системы (для СПЭМ особенно по точности преобразования и пропускной способности передачи данных) в условиях эксплуатации.

- Функционально-логическая реализация схем системы (для СПЭМ особое внимание уделяется многофункциональным связям с абонентами).

- Разработка способов и схем защиты от отказов.

- Макетирование и проверка работоспособности в условиях эксплуатации узлов (для СПЭМ особенно схем преобразования информации и сопряжения с магистралями).

- Конструктивно-технологическая проработка компоновки и трассировки устройств и узлов (для СПЭМ, в частности, с учетом влияния конструкции на точностные, временные и надежностные характеристики системы обмена).

- Разработка основных элементов ПО.

- Детальная схемотехническая и конструкторско-технологическая проработки системы с учетом защиты от отказов и обеспечения заданных характеристик.

- Испытания конструкции с оценкой ее влияния на точностные, временные и надежностные характеристики.

- Использование имитатора элементов системы при оценке основных характеристик на соответствие комплексу требований технического задания. 
При поэтапном проектировании после окончания каждого этапа выполняется оценка основных полученных результатов путем сравнения их с требуемыми по техническому заданию (ТЗ). На этапе разработки технического предложения, как правило, оцениваются параметры обслуживания выбранной структуры. По итогам эскизного проекта определяются основные характеристики функционально-логической схемы СПЭМ. Конструкторские, технологические решения, принципиальные электрические схемы системы позволяют на этапе разработки технического проекта получить все характеристики подсистемы обмена информацией в СПЭМ. На этапе испытаний осуществляется окончательная проверка принятых решений и происходит определение точных значений параметров СПЭМ в условиях эксплуатации.

После каждой оценки на этапах проектирования может быть выработана рекомендация корректировки ТЗ, необходимость исполнения которой решается дополнительно. При проведении оценок и составлении по ним рекомендаций дальнейших действий непосредственно учитывается специфика содержания работ на этапах проектирования СПЭМ.

\section{5. Результаты экспертного анализа ОСРВ для СПЭМ}

После анализа особенностей эксплуатации СПЭМ и анализа требований можно переходить к этапу экспертного анализа для выбора ОС реального времени для аппаратнопрограммной поддержки технологий экологического мониторинга [16-20].

\subsection{Phar Lap ETS $®$}

Phar Lap ETS - это оптимальное решение в реальном времени для устройств, основанных на архитектуре Intelx86. ETS обеспечивает простоту использования и предсказуемую производительность, в сочетании с высокой надежностью. ETS используется в мультимедийных потоковых решениях, субмикронном сканирование систем, системах определения местоположения морских судов и радиочастотной идентификации.

ETS - это высокопроизводительная микроядерная операционная система реального времени с оптимальным операционным следом 88k. Разработчики могут установить, настроить и начать разработку на ETS в течение 2-4 часов. Комплект разработки программного обеспечения ETS (SDK) обеспечивает набор инструментов, которые легко интегрируются в стандартную среду разработки Microsoft Visual Studio.

ETS Visual System Builder позволяет разработчикам систем выбирать компоненты ядра, эффективно строящие систему снизу вверх.

Поскольку ETS была разработана как совместимая с Win32 API операционная система, все стандартные соглашения Windows поддерживаются. Это включает в себя API, управление памятью, мьютексы и семафоры, к которым привыкли разработчики в Windows. В дополнение 
к производительности, ETS упрощает разработку благодаря обширному количеству инструментов, разработанных и оптимизированных для представления всеобъемлющей и подробной информации для инженера-программиста, чтобы быстро и точно решать проблемы по мере их возникновения в процессе разработки.

Архитектура ядра ETS обеспечивает полную гибкость в реализации, так как позволяет системным архитекторам использовать монолитное или разделенное ядро/приложение реализация. Возможность разделения приложения ETS позволяет обновлять на месте либо ядро, либо приложение.

Ядро ETS разработано на основе высокоскоростного планировщика, который использует как упреждающий, так и раундробиновые алгоритмы. ETS поддерживает неограниченное количество потоков и обеспечивает точный контроль над приложения с 256 уровнями назначаемого приоритета потока. Планировщик гарантирует, что критическое переключение контекста потока и переход к потокам с более высоким приоритетом происходят в течение 500 наносекунд в диапазоне менее 2 микросекунд. Это единственная операционная система реального времени, которая поддерживает надежное подмножество Win32 API и стандартную модель DLL. Эти возможности упрощают миграцию приложений с кодом на основе Windows и позволяет разрабатывать приложения ETS по частям с минимальным объемом памяти. Точное выполнение событий имеет решающее значение в системе реального времени. ETS обеспечивает полную гибкость разработчику, чтобы определить соответствующее разрешение таймера для своей системы. Значения интервалов таймера могут быть установлены между 1 микросекундами и 18,2 миллисекундами.

Важно, что ETS предоставляет разработчику высоко функциональную основу для построения систем, в том числе, встроенных систем, работающих в реальном времени ТСР / IP и USB 1.1 и 2.0. Сложные пользовательские интерфейсы и веб-службы полностью поддерживаются с помощью включенного графического пакета и интегрированного вебинтерфейса.

Разработка ПО с использованием ETS обеспечивает учет потребностей разработчика программного обеспечения. Система предоставляет комплексный набор инструментов, которые легко интегрируются в Microsoft IDE-Visual Studio. Эти инструменты обеспечивают инженер-программисту возможность интерактивного просмотра приложения в режиме реального времени, чтобы понять взаимодействие между оборудованием, ядром ETS и приложением ETS для отладки и анализа функционирования АПК системы экологического мониторинга. 


\subsection{VX Works}

OCPB VxWorks - это детерминированная, высокопроизводительная ОСРВ, которая устанавливает стандарт для масштабируемой, ориентированной на будущее, безопасной и надежной операционной среды для критически важных устройств и систем, которые должны соответствовать самым высоким стандартам.

Система VxWorks в режиме реального времени идеально подходит для жестких встроенных приложений реального времени, потому что это детерминированная, основанная на приоритетах, превентивная ОСРВ с низкой задержкой и минимальным дрожанием. В дополнение к стандартному вытеснению, VxWorks может обеспечивать режим, когда приложения, критичные для безопасности и времени, получали заданное количество циклов центрального процессора (ЦП) через различные формы планирования, а также разделение времени и пространства. Это также обеспечивает гибкость функций, необходимых для технологических процессов в различных отраслях промышленности.

Поскольку новые функции добавляются в VxWorks, и совместимость функций различных версий ПО всегда достаточно высока, то использование данной ОСРВ в СПЭМ весьма перспективно. Совместимость позволяет разработчикам воспользоваться последние инновации VxWorks, позволяющие им быстро добавлять новые функции и обновления с минимальным повторным тестированием всей системы, тем самым экономя время и затраты на проект.

Система имеет широкие возможности подключения и связи. Важно, что VxWorks имеeт надежные стеки IPv4 и IPv6, которые также поддерживают сети, чувствительные ко времени (TSN). Система гарантирует связь в реальном времени и доставку пакетов в течение ограниченного времени или задержки в коммутируемой сети Ethernet. VxWorks поддерживает инновационные промышленные приложения, включая, но не ограничиваясь, ОРС Unified Architecture (OPC UA); SocketCAN используемый в автомобильных приложениях; и хост, таргет и on-the-go (OTG) USB.

Имеется широкая поддержка многоядерных и многопроцессорных систем. Система VxWorks поддерживает 32- и 64-разрядные а также многоядерные процессоры, включая Intel®, Arm®, Power Architecture ${ }^{\circledR}$ и RISC-V. Такая всесторонняя поддержка многоядерных процессоров позволяет конфигурировать ОСРВ для асимметричной многопроцессорной обработки (AMP) и симметричной многопроцессорной обработки (SMP) с привязкой к процессору для связанной многопроцессорной обработки (BMP).

Благодаря совместной разработке с партнерами по экосистеме, VxWorks имеет самый обширный список пакетов поддержки плат в индустрии встроенного программного обеспечения. Осуществляется раннее создание прототипа, экономия средств и гибкость 
выбора. Решение проблемы для быстрого создания прототипов на недорогом оборудовании VxWorks предлагает BSP с открытым исходным кодом на GitHub, начиная с поддержки Raspberry Pi.

Важным для разработчика является надежность и настройка системы. Модульность VxWorks позволяет легко выбирать и адаптировать возможности по мере необходимости, замена модулей только по мере необходимости.

Высокий уровень виртуализации VxWorks обеспечивает эффективную, практически естественную производительность в виртуализированных средах, таких как платформа виртуализации Wind River® Helix ${ }^{\mathrm{TM}}$ или другие популярные гипервизоры, включая QEMU, VMware и KVM. Платформа Helix, которая имеет встроенный сертифицированный гипервизор, который может сэкономить время и усилия по сравнению с другими гипервизорами, которые непросто сертифицировать, также будут запускать VxWorks с другими операционными системами.

Отказоустойчивая файловая система VxWorks поддерживает высоконадежный файл Wind River. Имеется система (HRFS) для отказоустойчивости и восстановления операций в случае системной ошибки и завершение работы, а также FAT-совместимая файловая система dosFS.

\subsection{NI Linux Real-Time}

Компания National Instruments разработала ОС реального времени на основе Linux (OCPB), которая называется NI Linux Real-Time [20]. Уровень разработки высок благодаря многолетним исследованиям и разработкам, сотрудничеству с сообществом разработчиков программного обеспечения с открытым исходным кодом и участию партнеров. Эта ОСРВ доступна на некоторых аппаратных средствах NI, которые будут описаны ниже. NI Linux RealTime полностью поддерживается средой разработки NI LabVIEW с модулем реального времени LabVIEW.

Linux давно используется во встраиваемых системах в значительной степени потому, что это система бесплатна, с открытым исходным кодом, а также развивается и поддерживается сообществом разработчиков. При раннем использовании разработчики встроенного программного обеспечения пришли к выводу, что Linux не может быть OCPB, и с течением времени разработали множество гибридных подходов, которые объединяют Linux с выделенной ОСРВ для использования во встроенных приложениях.

По мере того, как использование Linux в разработке встроенных систем стало более зрелым, разработчики также добавили функции для улучшения детерминизма самого ядра Linux. Совсем недавно набор патчей PREEMPT_RT, являющийся результатом усилий по 
усовершенствованию ядра Linux, все чаще становится общепризнанным подходом к достижению реального результата в реальном времени с Linux.

Остановимся на преимуществах NI Linux при работе в реальном времени. Исторически производительность в реальном времени достигалась за счет удобства использования. Важно, что для NI Linux Real-Time, преимущества и удобство использования, которые предлагает Linux, переносятся и в саму OC, так как большая часть преимуществ возникает из-за ядра Linux, которое не нарушается. Таким образом, NI Linux Real-Time предлагает привлекательную производительность в режиме реального времени наравне с выделенными ранее ОСРВ, но без ущерба для удобства использования. Примером повышенного удобства использования, предоставляемого RTOS, является проверенный и стабильный сетевой стек, заимствованный из универсальной Linux. Благодаря улучшенному сетевому стеку целевые объекты реального времени, поддерживающие эту ОСРВ, могут поддерживать настоящие сетевые интерфейсные карты с двумя DHCP-серверами (NIC) и не требуют перезагрузки для применения изменений в настройках сети или времени, а также предлагают более широкую поддержку протоколов связи, таких как IPv6 и SNMP. Еще одним убедительным примером является поддержка дисплея для локальных HMI, которые NI Linux Real-Time предлагает в новом контроллере производительности CompactRIO.

Кроме того, поскольку Linux гораздо более распространен, чем даже самая популярная на рынке специализированная ОСРВ, данная ОСРВ предлагает гораздо более богатую экосистему и инструментарий, а также значительную базу опытных пользователей, способных использовать потенциал ОСРВ. При использовании режима реального времени, который поддерживается OC NI Linux Real-Time, у нас есть большая свобода дополнять функциональность нашего решения элементами экосистемы Linux. Можно также воспользоваться обширной базой опытных пользователей Linux. Отметим, что NI Linux RealTime расширяет возможности пользователей. От более простого добавления поддержки стороннего периферийного оборудования, такого как специализированные датчики, до гораздо более простой интеграции кода С / C ++.

Помимо удобства использования и экосистемы, в отличие от многих выделенных OCPB, NI Linux Real-Time является настоящей двухрежимной OC. В качестве двухрежимной OC NI Linux Real-Time предлагает значительно более устойчивую работу. В случае сбоя приложения система продолжает работать и может восстановиться после сбоя приложения без значительных потерь. NI Linux Real-Time также поддерживает настоящую многозадачность, позволяя запускать несколько программ параллельно. Например, можно запустить базу данных непосредственно на объекты, работающие в реальном времени, вместе с приложением LabVIEW Real-Time. 
И наконец, опираясь на Linux, эта ОСРВ предлагает значительно улучшенную возможность управления учетными записями пользователей и разрешениями файловой системы пользователей. Активность пользователей можно легче регистрировать на встроенных устройствах, которые поддерживают NI Linux Real-Time. Кроме того, могут быть реализованы такие функции безопасности, как VPN и брандмауэр, что исключает необходимость в дополнительном внешнем оборудовании исключительно для повышения безопасности сети.

NI Linux Real-Time обеспечивает производительность в реальном времени наравне с выделенными OCPB, предлагаемыми для целей CompactRIO текущего поколения. Джиттер целей NI Linux Real-Time имеет тот же порядок величины, что и джиттер в современных системах реального времени, предоставляемых NI. Что касается реальных приложений управления и потоковой передачи, то CompactRIO на основе реального времени NI Linux предлагает значительное увеличение производительности, поскольку циклы управления и потоковой передачи работают с более высокой частотой циклов при более низкой загрузке процессора.

\section{6. Заключение}

Таким образом, при выборе ОСРВ для СПЭМ следует четко представлять круг выполняемых проектных работ на соответствующих этапах и уровнях с использованием определенных методов проектирования СПЭМ и специализированных датчиков.

Метод сравнительного анализа позволяет определить допустимый вариант реализации OCPB, но имеет основные недостатки: в большинстве случаев на практике решение принимается по совокупности показателей качества, учитывающей технические характеристики ОС и аналогичные параметры класса таких систем, а по конкретным характеристикам. Это, как правило, осуществляется неавтоматизированным способом, что снижает эффективность разработки. Так как при этом на каждом шаге принятия решений по определенному параметру выбирается группа вариантов ОС для дальнейшего рассмотрения, то имеется возможность потери варианта реализации ОСРВ, наилучшего по критерию оценки следующих этапов анализа. Этот способ не позволяет создавать новые технические решения, поскольку ориентирован на оценку эвристически заданного множества конкурирующих вариантов. Отмеченный недостаток сравнительного анализа исключается при использовании для проектирования методов синтеза.

\section{Список литературы}

[1] Припутнев, Д.А., Экологический мониторинг окружающей среды / Д.А. Припутнев, И.Н. Мальцев, В.И. Лукьяненко, А.М. Чуйков // Проблемы обеспечения безопасности 
при ликвидации последствий чрезвычайных ситуаций. - 2015. - №1. - С. 182-185.

[2] Воронич, С.С. Оперативный экологический контроль атмосферных загрязнений локальных урбанизированных территорий / С.С. Воронич, А.Г. Хлопаев // Вестник ВолГУ. Серия 3: Экономика. Экология. - 2012. - №2. - С. 205-213.

[3] Анопченко, Л.Ю., Использование различных методов для экологического мониторинга атмосферного воздуха / Л.Ю. Анопченко, А.Ю. Луговская // Интерэкспо Гео-Сибирь. - 2014. - №2. - C. 84-88.

[4] Мазулина, О.В. Экологический мониторинг атмосферного воздуха / О В. Мазулина, Я.А. Полонский // «Инновации в науке»: материалы IX международной заочной научно-практической конференции. - Новосибирск: Сибирская ассоциация консультантов, 2012. - 102 с.

[5] Креймер, М.А. Климат и прогноз загрязнения атмосферного воздуха в городе / М.А. Креймер ／/ Интерэкспо ГЕО-Сибирь-2013. Междунар. науч. конф. «Дистанционные методы зондирования Земли и фотограмметрия, мониторинг окружающей среды, геоэкология» : сб. материалов в 2 т. (Новосибирск, 15-26 апреля 2013 г.). - Новосибирск: СГГА, 2013. - Т. 2. - 116-122 с.

[6] Путивцева, Н.П. Автоматизированная система экологического мониторинга / Н.П. Путивцева, К.В. Наливко // Проблемы Науки. - 2013. - №4(18). - С. 22-23.

[7] Пронин, Е.Г. Проектирование бортовых систем обмена информации. / Е.Г. Пронин, О.В. Могуева. - М.: Радио и связь, 1989. - 240 с.

[8] Ковалев, И.В. Математическое моделирование и алгоритмизация функций мониторинга технологических процессов на основе многоточечных измерительных систем / И.В. Ковалев, Д.И. Ковалев, В.В. Лосев, М.В. Сарамуд, Я.А. Тынченко // Современные наукоемкие технологии. - 2021. - № 6-1. - С. 29-38.

[9] Гофман, П.М., Отечественные стационарные системы виброконтроля и механических перемещений для питательных электронасосов тепловых электростанций / П.М. Гофман, И.В. Ковалев, Я.А. Тынченко, И.И. Кирьянов, В.В. Колесник // Промышленные АСУ и контроллеры. - 2019. - № 5. - С. 55-72.

[10] Колесник, В.В. Отечественные акселерометры для контроля абсолютной вибрации турбоагрегатов средней и большой мощности Приборы и системы / В.В. Колесник, С.А. Матвеев, П.М. Гофман, И.В. Ковалев, К.К. Першакова // Управление, контроль, диагностика. - 2018. - № 4. - С. 29-39.

[11] Ковалев, И.В. Мультиверсионная технология повышения достоверности измеряемых параметров производственно-экологического мониторинга / И.В. Ковалев, Д.И. Ковалев, М.В. Сарамуд, А.А. Ворошилова // Приборы. - 2018. - № 12(222). - С. 
48-52.

[12] Ковалев, И.В. Анализ технологического оборудования систем автоматизированного мониторинга дымовых газов тепловых электростанций / И.В. Ковалев, Д.И. Ковалев, В.В. Колесник, В.В. Лосев, М.В. Карасева // Сибирский журнал науки и технологий. - 2018. - T. 19. - № 4. - C. 683-690.

[13] Калинин, А.О. Методика расчета временных характеристик элементов автоматизированной системы управления на примере замкнутого контура регулирования давления на участке трубопровода под управлением контроллера "ОВЕН ПЛК100 220" / А.О. Калинин, М.В. Посконин, М.В. Сарамуд, В.В. Лосев, И.В. Ковалев // Сибирский журнал науки и технологий. - 2017. - Т. 18. - № 2. - С. 387-395.

[14] Гофман, П.М. Отечественные интеллектуальные бесконтактные реверсивные пускатели для асутп теплоэлектростанций средней мощности / П.М. Гофман, В.В. Колесник, И.В. Ковалев, К.К. Першакова // Промышленные АСУ и контроллеры. - 2016. - № 5. - C. 35-45.

[15] Гофман, П.М. Отечественные автоматизированные системы измерения абсолютной вибрации турбоагрегатов теплоэлектростанций / П.М. Гофман, В.В. Колесник, И.В. Ковалев, В.А. Драчёв, К.К. Першакова // Промышленные АСУ и контроллеры. - 2016. - № 12. - С. 20-35.

[16] Kovalev, I.V. Analysis of RTOS for hardware and software support of environmental monitoring technologies / I.V. Kovalev, D.I. Kovalev, A.S. Kuznetsov // IOP Conf. Series: Earth and Environmental Science. - 2020. - № 548032044.

[17] Рубанов, В.В. Разработка ОС реального времени для цифрового сигнального процессора / В.В. Рубанов, К.А. Власов // Труды ИСП РАН. - 2007. - №2. - С. 165-178.

[18] Касьянова, Е.Н. Особенности использования операционной системы QNX / Е.Н. Касьянова, О.А. Вдовыкина // Интеллектуальный потенциал XXI века: ступени познания. - 2012. - №13. - С. 69-72.

[19] Чуфырев, А.Е. Технический обзор особенностей операционной системы реального времени ti-rtos / А.Е. Чуфырев, В.А. Устюгов // Juvenis scientia. - 2016. - №1. - С. 37.

[20] Мороз, O.B. Принципы применения Linux в качестве операционной системы реального времени / О.В. Мороз, С.Н. Попов // Новые информационные технологии в автоматизированных системах. - 2012. - №15. - С. 37-41. 